\title{
Geo-environmental assessment of hydrocarbon contaminated sites in parts of central swamp depobelt, Eastern Niger delta
}

\begin{abstract}
The environmental degradation resulting from hydrocarbon exploration and production has diverse health and socioeconomic implications on ecosystem functionality and a major concern to all stakeholders globally and the Niger Delta in particular. Effective mitigation of these impacts is a site specific science and is dependent on accurate determination of extent of spread and prediction of contaminant behaviour. This study evaluated soil type, grain-size distribution, contaminant concentration, migration pathways and their effects on the environment. Soil and groundwater samples were collectedand analysed in the study area using standard methods. Results indicate that soil type is predominantly fine silty sands to fine gravel with permeabilities between $1.36 \times 10-4$ to $1.58 \times 10-4 \mathrm{md}$. Electrical conductivity values ranged between $38.6-$ $258.31 \mu \mathrm{S} / \mathrm{cm}$, Total Dissolved Solids (TDS) values between $64.5 \mathrm{mg} / \mathrm{L}-220.2 \mathrm{mg} / \mathrm{L}$, Total Suspended Solids values ranged from $24.0 \mathrm{mg} / \mathrm{L}-187 \mathrm{mg} / \mathrm{L}$, while heavy metals values were within acceptable limits. Atterberg limits for soils were higher at impacted sites as against the control sites. Collaborated effort by government, operating oil companies and other stakeholders are necessary in achieving a sustainable pollution management in the area.
\end{abstract}

Keywords: contamination, environmental degradation, hhydrocarbon exploration, pollution, swam
Volume 2 Issue 3 - 2017

\author{
Nwankwoala HO, Mzaga TM \\ Department of Geology, University of Port Harcourt, Nigeria \\ Correspondence: Nwankwoala HO, Department of Geology, \\ University of Port Harcourt, Nigeria, \\ Emailnwankwoala_ho@yahoo.com
}

Received: February 17, 2017 | Published: May 23, 2017

\section{Introduction}

The flow dynamics and fate of hydrocarbon contaminants once released into the environment are unknown. ${ }^{1}$ The scientific understanding of the environmental effects and behaviour of spilled crude oil in the environments is very important in identifying effective strategies for spill preparedness, spill emergency response and environmental remediation. A very sensitive ecosystem made up wetlands and a large drainage basin, the Niger Delta ecosystem supports all life forms (fauna and flora)", variety of crops, trees and aquatic life than any other ecosystems in west Africa". ${ }^{2}$ The terrain cut across land, swamp and mangrove ecosystems with generally flat plains of complex network of interconnected creeks, creeklets, rivers and tributaries with gentle slope into the ocean. ${ }^{2}$ Oil exploration and production has precipitated a wide range of environmental problems associated with its activities. Crude oil releases into the environment is necessitated through a variety of means such as leaks from storage, production, processing and pipeline facilities, accidental releases, byproducts of industrial activity, crude oil theft and wilful damage to oil installations. Contamination of the environment has significant impact on human and environmental health worldwide. ${ }^{3}$ The chemicals and products commonly released into the environment have associated toxic or carcinogenic properties from acute and/or chronic exposure to humans and other organisms. ${ }^{4}$ An understanding of the chemistry contributes immensely to predicting a substance's fate and transport in order to develop appropriate mitigation and remediation strategies. ${ }^{5}$ Depending on the type and nature of the environmental media, crude oil is released into the environment denatures the components of the environment. The degree of alteration depends on the nature of the component e.g. geology, composition and quantity of the spilled product. ${ }^{6}$ Smaller volumes of volatile hydrocarbon products spilled on dry sandy soil environments has least impact, the hydrocarbons evaporate easily and fast, causing little or no damage the soil (physical or chemical). When heavy crude oil products is released onto soil such as clay, the oil adhere onto the soil grains and can remain there for longer periods, and cause alteration to soil permeability, alter its toxicity levels and reduce or destroy soil quality. In this case such soil can become a secondary source of pollution. The dominant crude oil types produced in Nigeria, contain aliphatic fractions $>16-44$ and $>12-16$, a quality that enables easy infiltration into porous media within a very short period (SPDC Risk Based Methodology (RBM), 2002). Spill incidents accompanied by fire outbreak usually leads to further destruction of the soil fabric, mineralogical alterations, mass loss, alteration of soil permeability (Zhilms, et al., 2013) thereby enhancing contaminant migration into the groundwater aquifers. At artisanal refining sites, the heat from the distilleries affects the soil permeability, mass loss, particle size distribution and mineralogy. This enhances contaminant infiltration into the groundwater aquifers. ${ }^{7}$ The contaminated Groundwater unlike surface water contamination is considered a more difficult problem because of the difficulty in its early detection and skill required for prediction of its rate and path of movement ${ }^{2}$ and the extent of the impact. Effective clean up and Remediation of these contaminated sites can only be achieved with adequate knowledge of the interplay of site specific factors such as geology, nature of the contaminant, pathway receptors linkages , toxicity levels and deployment of appropriate contamination management techniques and legislation. This study therefore is aimed at determining the effect of contamination on the physical soil characteristics, assessment of the contamination and toxicity levels 
of hydrocarbon contaminants at the sites, evaluating the hydrocarbon contaminant migration pathway and groundwater flow direction as well as suggest sustainable management options.

\section{The study location}

The area of study is situated between latitudes 4039'43.6"' 4046'19.6" and longitude 707'8.8"-7016'38.3" (Figure 1). The land cover of this area consists of agricultural lands, natural vegetation and tree crop plantations, generally mangroves, freshwater swamp forests and rainforests. ${ }^{8}$ The topography is essentially flat and slopes gently seawards. The area is poorly drained and crisscrossed by network of tributaries Perennial inundation of the areas due to high annual rainfall cause overflow of rivers and their banks. ${ }^{9,10}$

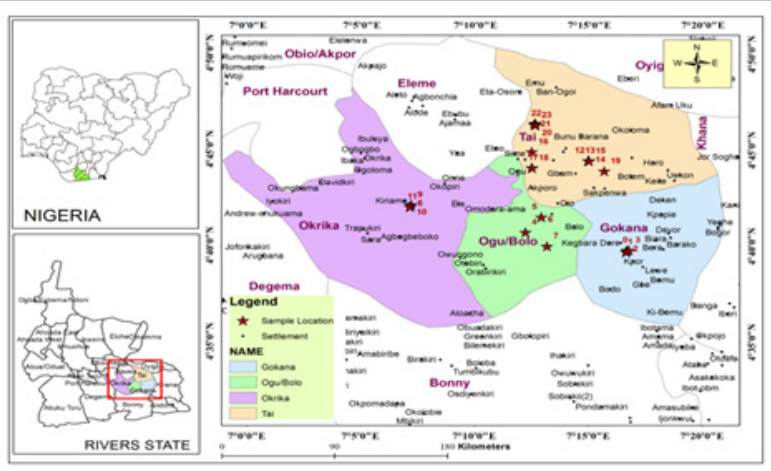

Figure I Location map of study area showing sample locations.

\section{Geology of the study area}

The area of study is predominantly made up of soils ranging from fine silts, sand and fine gravel types. The geology (Figure 2) generally shows a coarsing upward sequence indicating a regressive deposition which is in line with the geology of Niger Delta., ${ }^{9,11}$ The surface elevation of the area is a gentle slope towards the west. The upper cretaceous deposits of the Niger delta are fluvial and marine in nature formed about 50million years ago. An accumulation of over $12,000 \mathrm{~m}^{3}$ of marine deltaic sediments overtime transported mainly from river Niger and its tributaries.

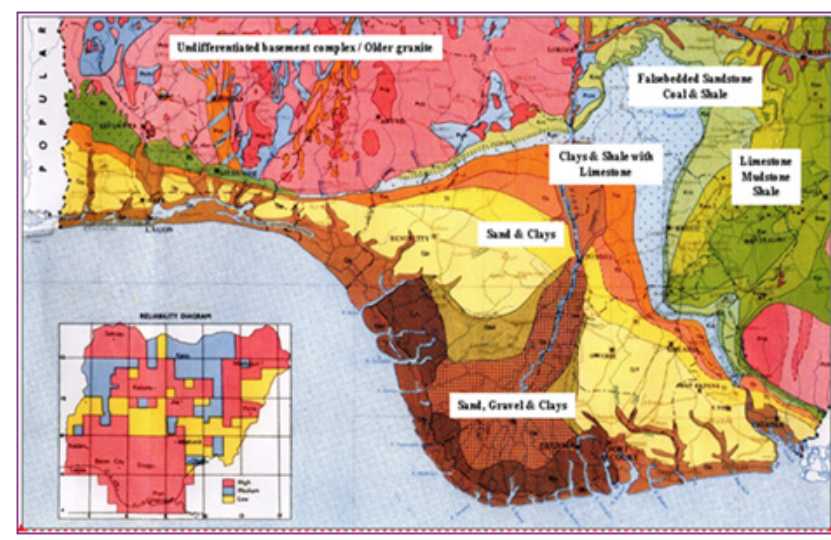

Figure 2 Geological map of Niger Delta(SPDC, 2002).

It is characterized by three major lithofacies:

(i) varying thickness of marine shales and claystones at the base;

(ii) Alternating claystones, sandstones and silstones with a progressively upward increase of sand percentage;

(iii) Topmost alluvial sands. Benin Formation, the uppermost geological layer is made up of multiple layers of sand, clays, conglomerate, lignite and peat of various thickness and textures and covered by thick overburden soil. "The deltaic plain is flat lying at about $40 \mathrm{~m}$ above sea level towards the interior, and less than $8 \mathrm{~m}$ above sea level on approaching the coast". 9,12 The area is a flat lowlying plains, crisscrossed by creeklets, riversand its tributaries that drain the entire area to the sea.

The Niger Delta is periodically exposed to inundation especially during the heights of rainfall when the plains get flooded and creeks, creeklets rivers and tributaries are overflow their banks with a charactristic negligible surface flow. ${ }^{13}$

Akpokodje, (1987) described the type of soils encountered inland from the Atlantic Ocean as:

i) coastal beach ridge and sand;

ii) dark organic peat clay;

iii) light grey fine sand to silt clay;

iv) brownish sandy clay; and reddish-brown sandy clay loam.

However, clay and loamy soils separate the topsoil from the aquifer, but the clay is no longer continuous as previously thought according to the United Nations Environmental ProgrammeAssessment Report ofOgoniland 2011..$^{10,14}$

\section{Hydrogeology of the study area}

Many researchers such as Nwankwoala and Ngah; Ngah and Nwankwoala; Etu-Efeotor and Akpokodje(1990); Amadi et al.; EtuEfeotor; Weber and Dakouru ${ }^{2,9,15-19}$ have studied the hydrogeology of the area at different times and have described the Benin Formation as a highest yielding water bearing zone of the area. Overlying the 40m-150m thick Quaternary deposits, the Benin Formation consists of sequences of sands and silty clay alternating which later become increasingly prominent seawards. ${ }^{9,17}$ Based on strata logs in the area, described the aquifer in the area as a stack of alternating aquifers lying upon each other in a multiple fashion such that the uppermost ones are mostly unconfined and underlain by the confined aquifers. The Niger Delta consists of three diachronous units, namely Akata (oldest), Agbada and Benin (youngest) formations. The Benin Formation (Oligocene to Recent) is about $2100 \mathrm{~m}$ thick at the basin centre and consists of medium to coarse grained sandstones, thin shales and gravels. ${ }^{19}$ The Niger Delta has spread across a number of ecological zones comprising sandy coastal barriers, brackish or saline mangrove, freshwater and seasonal swamp forests. The Niger Delta has two most important aquifers, Deltaic and Benin Formations. With a typically dendritic drainage network, this highly permeable sands of the Benin Formation allows easy infiltration of water to recharge the shallow aquifers. Nwankwoala et al. ${ }^{20}$ described the aquifers in this area as a set of multiple aquifer systems stacked on each other with the unconfined upper aquifers occurring at the top. ${ }^{21}$ Recharge to aquifers is direct from infiltration of rainfall, the annual total of which varies between $5000 \mathrm{~mm}$ at the coast to about $2540 \mathrm{~mm}$ landwards. Groundwater in the area occurs in shallow aquifers of predominantly continental deposits encountered at depths of between $45 \mathrm{~m}$ and $60 \mathrm{~m}$ (Figure 3 ). The lithology comprises a mixture of sand in a fining up sequence, gravel and clay. Well yield is excellent, with 
production rates of 20,000 litres/hour common and borehole success rate is usually high..$^{22,23}$ Surface water occurrence includes numerous networks of streams, creeks and rivers.

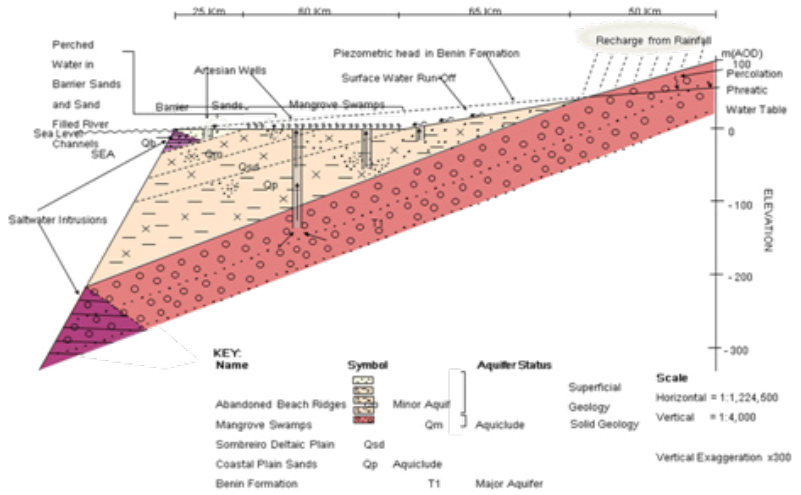

Figure 3 Hydrogeological features across the Niger Delta ( SPDC, 2002).

\section{Materials and methods}

Four (4) soil/sediment and two (2) groundwater samples were collected at each locality at potential contaminated sites and the control sample at clean site. Three (3) samples each were collected at each sample location at various depths $(1.0 \mathrm{~m}, 2.0 \mathrm{~m}$ and $3.0 \mathrm{~m}$ discrete samples) respectively. Location and sample point positions were gotten with use of a hand held Global Positioning System (GPS) device. Soil samples were collected from hand augered holes at depths of $1 \mathrm{~m}, 2 \mathrm{~m}$, and $3 \mathrm{~m}$ while manually operated drill was used to bore holes for groundwater sampling. Soil samples were duplicated for geochemical and geotechnical analysis each while water samples were collected for geochemical tests. In order to get accurate onsite data, portable handheld devices soil $\mathrm{pH}$ and soil moisture were used. The geographical position (Figure 4) of the sample point was established and read off using a GPS device and recorded. Discrete Soil Samples were collected using the grab method with aid of a hand auger and water samples were also collected from boreholes drilled using percussion drilling methods. Four Cable percussion boreholes were designed e.g. NOW-BH1, NOW-BH2, NOW-BH3 and NOW-CTR for drilling at the 4 locations: Nonwa, Sime, Norkpo and Bomu sites respectively to depths of $10 \mathrm{~m}$ below existing ground level (bgl). Standard cable percussion boring equipment was used to produce $150 \mathrm{~mm}$ diameter boreholes. Environmental samples were collected at $0-5 \mathrm{~m}$ and at every meter from $1-10 \mathrm{mbgl}$ during drilling to enable a comprehensive assessment of the strata encountered. Where present, the depth to the underlying groundwater was recorded upon encounter. The samples were then stored in an ice-packed cooler for transport to the laboratory for analysis. The analysis of chemicals of concern was in accordance with regulatory standard. Falling head tests were conducted on one of the cable percussion boreholes at the site to determine the permeability rate for the underlying soils. The permeability of the soil has been calculated in accordance with the guidelines presented in BS 5930:1999 (Hvorslev Method 1).The hydraulic conductivity rate for the sites were estimated from geotechnical data.

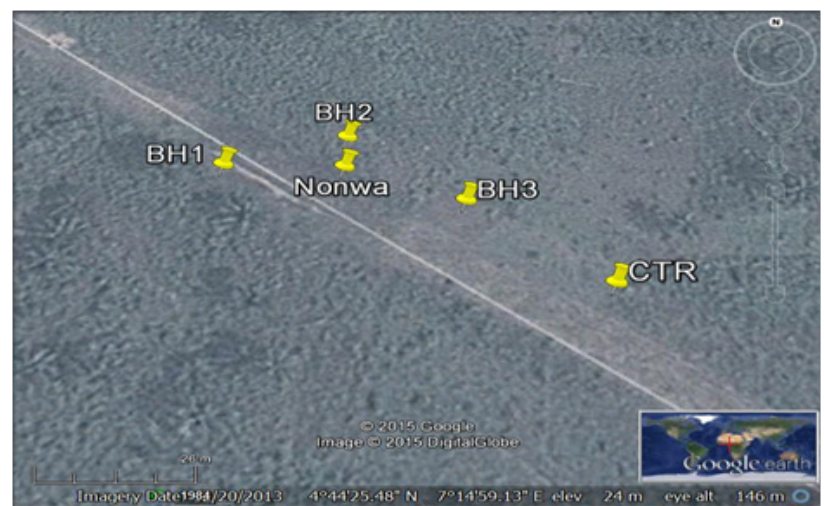

Figure 4 Typical Google map showing sampling locations.

\section{Discussion of results}

\section{Soil moisture content}

Soil moisture content value ranges from 5.2-97. 9 with spikes at Okrika OKR-CTRL and BM-CTRL probably due to shallow water table encountered at shallow depth of $2.0 \mathrm{~m}$, with an average moisture level of 20.08 and that of the control sites, 17. 85. The slight difference in moisture could have resulted from the introduction of the fourth phase on the soil structure which possibly gave rise to more water molecules adhering unto the soil grains and in effect creating an atmosphere for chemical reactions to take place in the soil. The variation also suggests higher water retention potential of contaminated soils against normal clean soil which enhance contaminant persistence in the soil. Table 1 show the variation of moisture across various sites and depths.

\section{Soil pH}

The soil $\mathrm{pH}$ values for control sites tend to normal range i.e. towards 7.0, while the values for impacted sites are slightly lower. Most samples had normal soil $\mathrm{pH}$ ranges except BM-SS1 which indicated acidic soil conditions with ranges of 4.5-5.8. At a petroleum impacted site as observed at $\mathrm{BH}-\mathrm{SS}$ (Table 2), $\mathrm{pH}$ readings tend to strong acidity probably due to history of prolonged hydrocarbon contamination at the site. Small changes in $\mathrm{pH}$ values can induce severe changes in the sensitive biochemical environment thereby altering biological and chemical processes. For example, a soil with pH 5 is 10 or 100 times more acidic than a soil with $\mathrm{pH} 6$ or 7 , respectively.

\section{Soil and groundwater chemical analysis}

Results revealedthat 54 and 18 soil and 10 groundwater samples respectively had TPH concentrations above the target levels of $50 \mathrm{mg} / \mathrm{kg}$. The control samples NOR-CTRL, NOW-CTRL, OKRCTRL all had TPH values above target level. The highest soil TPH was observed mostly at depths $1 \mathrm{~m}-3 \mathrm{~m}$ across the sites. Moderate concentrations were recorded at Ogu Bolo (OGB-SS2;OGBSS3), Okrika (OKR-SS2;OKR-SS3), Nonwa (NOW-BH1; NOW$\mathrm{BH} 3$ ),Norkpo (NOR-BHD,NOR-BHJ) and low values were observed at sample points BM-SS3,NOW-BH2, SIM-BH,SIM-BH2,SIM-BH3 and all the control points (Table 3).Tidal changes around Okrika and 
Ogu-Bolo have the potential to carry contaminants offsite to other areas in addition to overland flow and infiltration. A residual TPH concentration of $1,000 \mathrm{mg} / \mathrm{kg}$ is capable of contaminating water resources and puts the water resources in the study areas at great risk of contamination. Volatile Organic Compounds (VOCs) fractions i.e. the BTEX group are notorious contaminants of soil and groundwater. The amount of 'Total BTEX' is useful in risk ranking of sites and the need of remediation prioritization of such sites. Target concentration values for this chemical is $0.005 \mathrm{mg} / \mathrm{l}$. Stringent cleanup requirements for groundwater protection for benzene vary from $0.01 \mathrm{mg} / \mathrm{L}$ to $20 \mathrm{mg} / \mathrm{L}$. Depending on site conditions and location of the receptor, corresponding groundwater values range from 0.005 to $0.30 \mathrm{mg} / \mathrm{L} .^{24}$ Hydrocarbon contaminant concentrations across the sites were within regulatory standard at Bomu Manifold,Ogu-Bolo, Okrika and Norkpo whereas Nonwa and Sime have no significant concentrations. The concentration spike at Bomu manifold across all the depths investigated implied that the shallow water table at $3.0 \mathrm{~m}$ is vulnerable (Table 3).The concentration trends show significant values at all the impacted sites except Sime which has significantly low values at all the depths investigated. Groundwater samples analyzedindicated contaminant concentrations above EGASPIN target levels while 5 were below target. Site observations also showed free phase product observed at Nonwa (NOW-BH1,BH-2), Bomu (BM-BH1 and BM-
BH2).Thirteen (13) had exceeded EGASPIN Intervention values for $\mathrm{TPH}$ at $5 \mathrm{~m}, 7 \mathrm{~m}, 9 \mathrm{~m}$ and $10 \mathrm{~m}$ of $\mathrm{BH} 1 ; 9 \mathrm{~m}$ and $10 \mathrm{~m}$ in 2 Boreholes at Nonwa. Eighteen for VOC fractions (BTEX) in six boreholes at depths of $7-10 \mathrm{mbgl}$; Benzene at $7 \mathrm{~m}$ and $10 \mathrm{~m}$ in four boreholes and $10 \mathrm{~m}$ in three Boreholes at Nonwa and Sime; Xylene at $9 \mathrm{~m}$ and $10 \mathrm{~m}$ in Nonwa; The deep subsurface soil $(>7 \mathrm{mbgl})$ at the sites showed varying degree of hydrocarbon contamination. TPH is detected at shallow depth with visible free phase product was observed at Bomu, Nonwa and Norkpo while deeper contamination at depth $7 \mathrm{~m}-10 \mathrm{~m}$ bgl was observed at Nonwa and Sime with possibilities of hydrocarbon at deeper strata.

\section{Microbiological profiles of soil samples}

Hydrocarbon utilizing organism populations of soil samples are shown in Table 4 \& Table 5 Hydrocarbon utilizing microbial counts recorded at the sites show that hydrocarbon utilizing bacteria (HUB) populations are higher at the sites, with a highest value of $8.82 \times 10^{4} \mathrm{cfu} / \mathrm{g}^{-1}$ while hydrocarbon utilizing fungi (HUF) populations ranges recorded $5.1 \times 10^{4} \mathrm{cfu} / \mathrm{g}^{-1}$ and below across the sites. Higher populations of the bacteria over the fungi were generally higher in samples at depths $1 \mathrm{~m}$ across the sites but highest in samples BM-SS1, OGB-SS1, NOR-BHC and SIM-BH1. The highest count recorded at 2m was BM-SS1, BM-SS2, and BM-SS3.

Table I Results of on-site parameters

\begin{tabular}{|c|c|c|c|c|c|c|c|c|c|c|}
\hline \multirow{3}{*}{ Site name } & \multirow{3}{*}{ Sample ID } & \multicolumn{2}{|c|}{ GPS Co-ordinates } & \multirow{3}{*}{ Elevation } & \multicolumn{6}{|c|}{ Insitu parameters } \\
\hline & & \multirow{2}{*}{$\begin{array}{l}\text { Northing } \\
\text { latitude }\end{array}$} & \multirow{2}{*}{$\begin{array}{l}\text { Easting } \\
\text { latitude }\end{array}$} & & \multicolumn{3}{|c|}{ moisture content } & \multicolumn{3}{|l|}{$\mathbf{p H}$} \\
\hline & & & & & $1 \mathrm{~m}$ & $2 \mathrm{~m}$ & $3 \mathrm{~m}$ & $1 \mathrm{~m}$ & $2 \mathrm{~m}$ & $3 \mathrm{~m}$ \\
\hline \multirow{4}{*}{ Bomu Manifold } & $\mathrm{BM}^{-\mathrm{SS}_{1}}$ & 4.66294 & 7.27788 & $20.70 \mathrm{~m}$ & 18.67 & 18.7 & 29.21 & 4.6 & 4.5 & 5.8 \\
\hline & $\mathrm{BM}-\mathrm{SS}_{2}$ & 4.661526 & 7.277251 & $15.60 \mathrm{~m}$ & 25.04 & 15.19 & 21.32 & 6.6 & 6.55 & 6 \\
\hline & $\mathrm{BM}-\mathrm{SS}_{3}$ & 4.661585 & 7.2777208 & $17.90 \mathrm{~m}$ & 28.04 & 29.88 & 17.12 & 6.65 & 6 & 6.45 \\
\hline & BM-CTRL & 4.66294 & 7.279345 & $17.60 \mathrm{~m}$ & 15.2 & 72.9 & NS & 7 & 6.3 & NS \\
\hline \multirow{6}{*}{ Ogu Bolo } & $\mathrm{OGB}-\mathrm{SS}_{1}$ & 4.67842 & 7.20369 & $10.80 \mathrm{~m}$ & 19.6 & 18.7 & 29.21 & 6.65 & 6.6 & 5.8 \\
\hline & OGB-SS & 4.691593 & 7.215291 & $15.50 \mathrm{~m}$ & 25.04 & 15.19 & 21.32 & 6.55 & 6.55 & 6 \\
\hline & OGB- Control & 4.66636 & 7.219411 & $6.60 \mathrm{~m}$ & 5.2 & 6.4 & 6.8 & 6.8 & 6.7 & 6.5 \\
\hline & $\mathrm{OKR}_{-\mathrm{SS}_{1}}$ & 4.702188 & 7.119898 & $12.70 \mathrm{~m}$ & 18.67 & 18.7 & 29.21 & 6.6 & 6.6 & 5.8 \\
\hline & $\mathrm{OKR}_{-} \mathrm{SS}_{2}$ & 4.702108 & 7.119892 & $0.70 \mathrm{~m}$ & 25.04 & 15.19 & 21.32 & 6.5 & 6.55 & 6 \\
\hline & $\mathrm{OKR}_{-} \mathrm{SS}_{3}$ & 4.700406 & 7.11932 & $6.90 \mathrm{~m}$ & 28.04 & 29.88 & 17.12 & 6.65 & 6 & 6.45 \\
\hline
\end{tabular}

Okrika

$\begin{array}{lllllllll}\text { OKR-CTRL } & 4.70048 & 7.11891 & 9.50 \mathrm{~m} & 15.2 & 97.9 & \text { NS } & 6.2 & 6.9\end{array}$


Table Continued

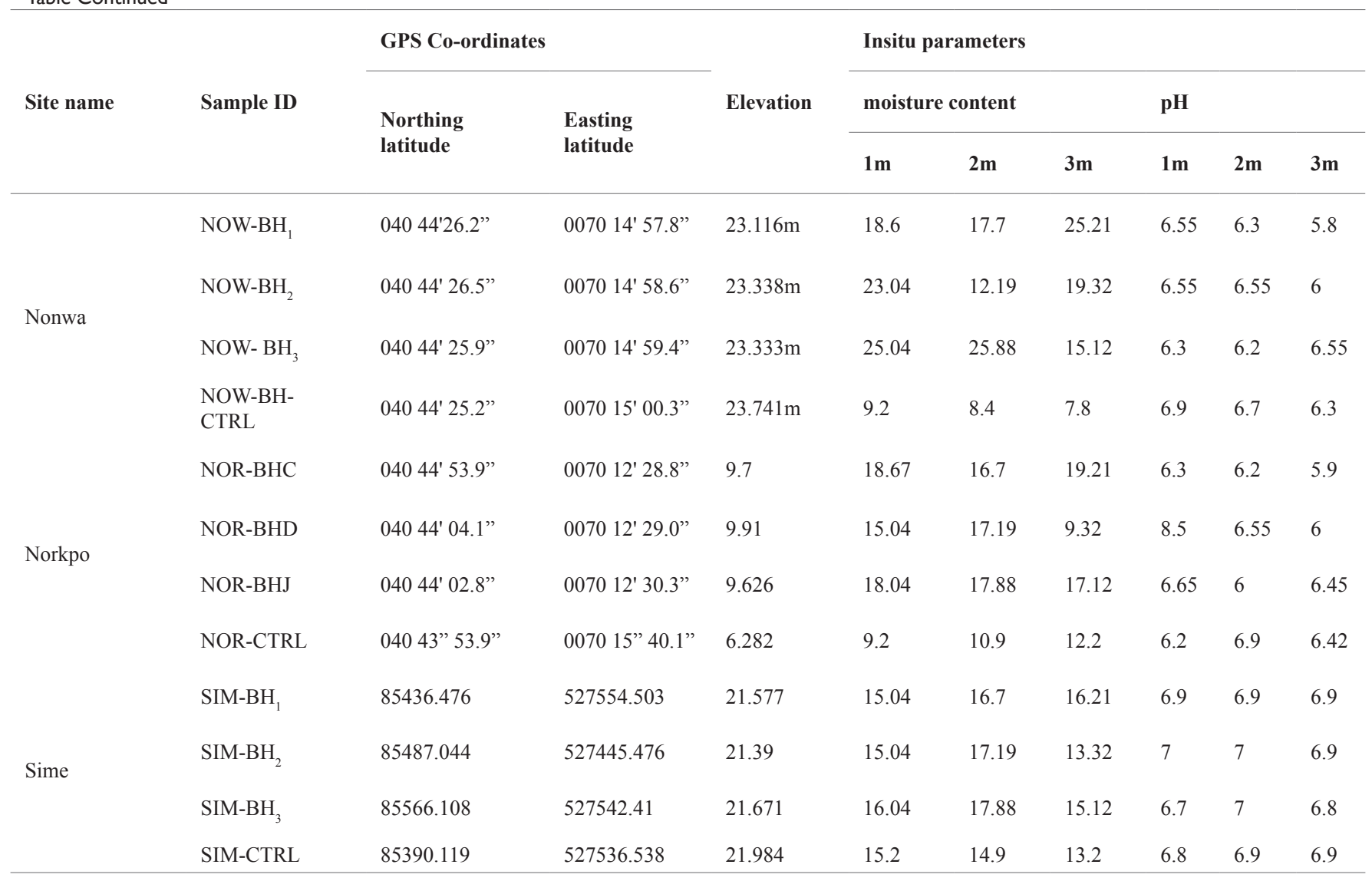

Table 2 Geochemical analysis results for soil

\begin{tabular}{|c|c|c|c|c|c|c|c|c|c|c|c|c|}
\hline \multirow{3}{*}{$\begin{array}{l}\text { Site } \\
\text { name }\end{array}$} & \multirow{3}{*}{$\begin{array}{l}\text { Sample } \\
\text { ID }\end{array}$} & \multicolumn{2}{|c|}{ GPS Co-ordinates } & \multicolumn{9}{|c|}{ Geochemical parameters } \\
\hline & & \multirow{2}{*}{$\begin{array}{l}\text { Northing } \\
\text { latitude }\end{array}$} & \multirow{2}{*}{$\begin{array}{l}\text { Easting } \\
\text { latitude }\end{array}$} & \multicolumn{3}{|c|}{ TPH (mg/kg) } & \multicolumn{3}{|c|}{ BTEX(mg/kg) } & \multicolumn{3}{|c|}{ PAH(mg/kg) } \\
\hline & & & & $1 \mathrm{~m}$ & $2 \mathrm{~m}$ & $3 \mathrm{~m}$ & $1 \mathrm{~m}$ & $2 m$ & $3 \mathrm{~m}$ & $1 \mathrm{~m}$ & $2 \mathrm{~m}$ & $3 m$ \\
\hline \multirow{4}{*}{$\begin{array}{l}\text { Bomu } \\
\text { Manifold }\end{array}$} & $\mathrm{BM}-\mathrm{SS}_{1}$ & 4.66294 & 7.27788 & $6,687.74$ & $6,417.21$ & $8,642.46$ & 118.23 & 118 & 112.5 & 6.76 & 23.5 & 12.6 \\
\hline & $\mathrm{BM}-\mathrm{SS}_{2}$ & 4.661526 & 7.277251 & 5,000 & $5,116.89$ & $5,958.70$ & 35.45 & 22.32 & 10.6 & 23.9 & 29.7 & 121.8 \\
\hline & $\mathrm{BM}-\mathrm{SS}_{3}$ & 4.661585 & 7.2777208 & 79.84 & $5,332.55$ & $4,795.00$ & 118.9 & 112.4 & 110.2 & 150.6 & $<0.001$ & $<0.001$ \\
\hline & BM-CTRL & 4.66294 & 7.279345 & 39.35 & 30.56 & 23.7 & $<0.001$ & $<0.001$ & $<0.001$ & $<0.001$ & $<0.001$ & $<0.001$ \\
\hline \multirow{4}{*}{ Ogu Bolo } & $\mathrm{OGB}-\mathrm{SS}_{1}$ & 4.67842 & 7.20369 & $12,303.42$ & $6,545.28$ & 2,546 & 40.01 & $<34.01$ & $<13.01$ & 58.8 & 30.001 & 23.7 \\
\hline & ${\mathrm{OGB}-\mathrm{SS}_{2}}_{2}$ & 4.691593 & 7.215291 & $4,736.92$ & $2,735.48$ & 3,332 & 54.06 & 30.06 & $<0.001$ & 113.6 & 112.01 & 22.34 \\
\hline & ${\mathrm{OGB}-\mathrm{SS}_{3}}_{2}$ & 4.691618 & 7.2153 & 2,543 & 1,554 & 1,657 & 117.01 & $<9.01$ & $<0.001$ & 112 & 89.65 & $<0.001$ \\
\hline & $\begin{array}{l}\text { OGB- } \\
\text { Control }\end{array}$ & 4.66636 & 7.219411 & 27.18 & 48.05 & 50.2 & $<0.001$ & $<0.001$ & $<0.001$ & $<0.001$ & $<0.001$ & $<0.001$ \\
\hline \multirow{4}{*}{ Okrika } & $\mathrm{OKR}_{-} \mathrm{SS}_{1}$ & 4.702188 & 7.119898 & $6,678.15$ & $3,545.28$ & 2,546 & 40.01 & $<34.01$ & $<13.01$ & 158.8 & 130 & 53.7 \\
\hline & $\mathrm{OKR}_{-} \mathrm{SS}_{2}$ & 4.702108 & 7.119892 & $2,154.40$ & $2,735.48$ & 2,332 & 5.06 & 33.06 & $<0.001$ & 213.6 & 72.01 & 22.34 \\
\hline & $\mathrm{OKR}_{-} \mathrm{SS}_{3}$ & 4.700406 & 7.11932 & 2,543 & 1,554 & 1,657 & 17.01 & 9.01 & $<0.001$ & 112 & 89.65 & 30.1 \\
\hline & $\begin{array}{l}\text { OKR- } \\
\text { CTRL }\end{array}$ & 4.70048 & 7.11891 & 857.7 & 248.05 & 150.2 & $<0.001$ & $<0.001$ & $<0.001$ & $<0.001$ & $<0.001$ & $<0.001$ \\
\hline
\end{tabular}


Table Continued

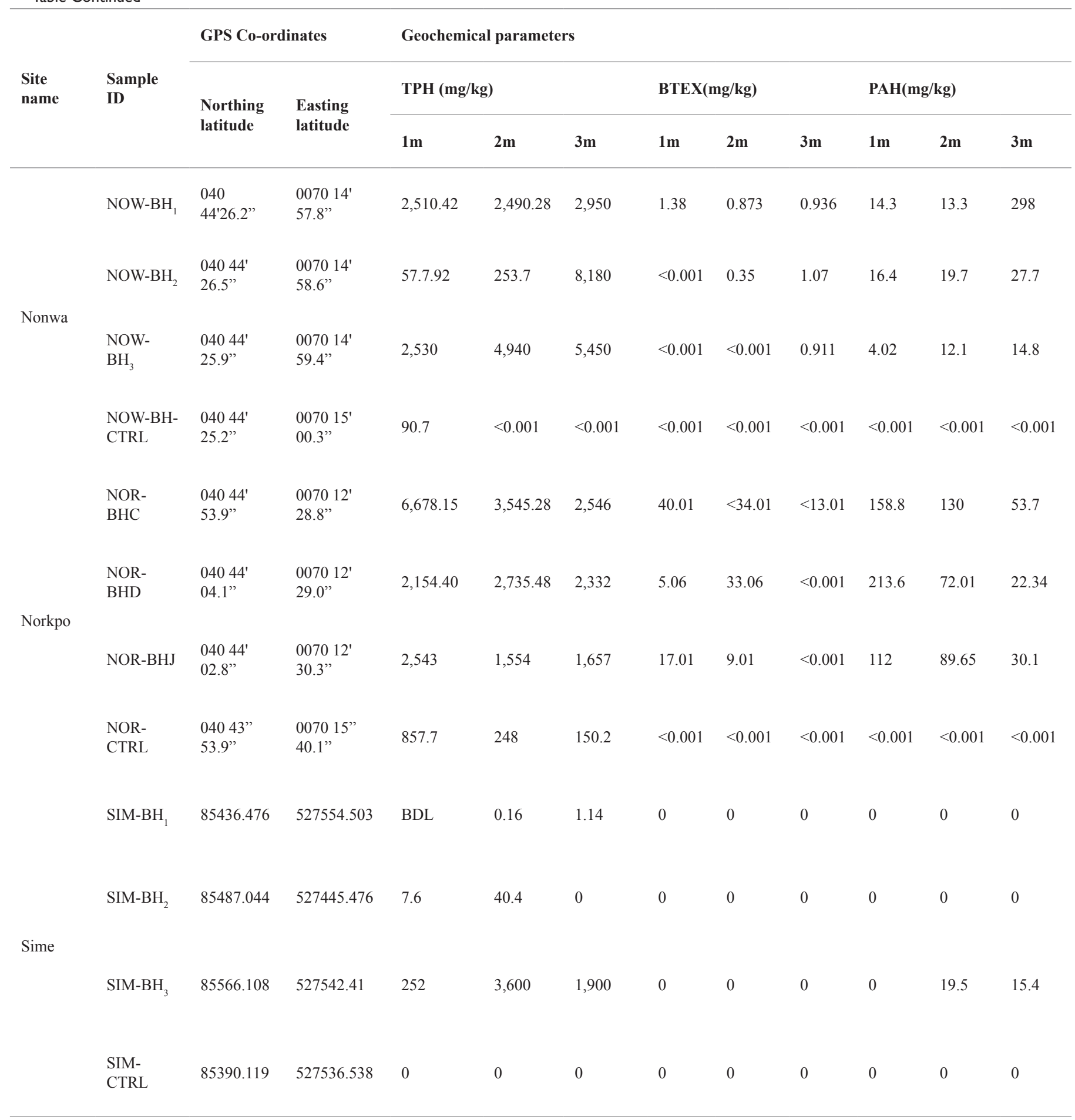


Table 3 Physical and chemical composition of groundwater samples

\begin{tabular}{|c|c|c|c|c|c|c|c|c|c|c|}
\hline Regulatory limits & & & & NORKPO & SIME & NONWA & BOMU & $\begin{array}{l}\text { OGU- } \\
\text { BOLO }\end{array}$ & OKRIK & \\
\hline $\begin{array}{l}\text { Analysis } \\
\text { parameters }\end{array}$ & $\begin{array}{l}\text { EGASPIN } \\
\text { Target } \\
\text { values }\end{array}$ & $\begin{array}{l}\text { WHO } \\
\text { Limit }\end{array}$ & UNITS & BH- NOR & $\begin{array}{l}\text { BH- } \\
\text { SIM }\end{array}$ & BH-NOW & BH-BM & BH-OGB & $\begin{array}{l}\text { BH- } \\
\text { OKR }\end{array}$ & Total \\
\hline ТPH & 50 & 50 & $\mu \mathrm{g} / \mathrm{L}$ & 345 & 1840000 & 381000 & 34000 & 1,200 & 760 & 2257305 \\
\hline PAH & 0.1 & 1 & $\mu \mathrm{g} / \mathrm{L}$ & BDL & 1010 & 2620 & 6.88 & BDL & $\mathrm{BDL}$ & 3636.88 \\
\hline Benzene & 0.2 & 0.2 & $\mu \mathrm{g} / \mathrm{L}$ & BDL & $<1$ & 2080 & 23 & 14 & 32 & 2149 \\
\hline Toluene & 0.2 & 0.2 & $\mu \mathrm{g} / \mathrm{L}$ & BDL & $<1$ & 3870 & $<1$ & $<1$ & $<1$ & 3870 \\
\hline Ethylbenzene & 0.2 & 0.2 & $\mu \mathrm{g} / \mathrm{L}$ & BDL & 24200 & 1170 & $<1$ & 21 & 56 & 25447 \\
\hline Xylene & 0.2 & 0.2 & $\mu \mathrm{g} / \mathrm{L}$ & BDL & 258000 & 8030 & $<1$ & $<1$ & $<1$ & 266030 \\
\hline $\mathrm{pH}$ & $6.5-8.5$ & 9.2 & N/A & 6.1 & 6.4 & 7 & 5.4 & 6.2 & 6.3 & 37.4 \\
\hline Temperature & 35 & 28 & $0 \mathrm{C}$ & 27 & 26.9 & 27.1 & 26.2 & 26.3 & 27.2 & 160.7 \\
\hline $\begin{array}{l}\text { Electrical } \\
\text { Conductivity }\end{array}$ & N/A & $0-40$ & $\mu \mathrm{S} / \mathrm{cm}$ & 258.31 & 98.5 & 143.3 & 121.7 & 124.85 & 38.6 & 785.26 \\
\hline $\begin{array}{l}\text { Total Dissolved } \\
\text { solids (TDS) }\end{array}$ & 800 & 1000 & $\mathrm{mg} / \mathrm{l}$ & 121.32 & 96.5 & 89.2 & 64.5 & 66.17 & 220.2 & 657.89 \\
\hline $\begin{array}{l}\text { Total suspended } \\
\text { solids (TSS) }\end{array}$ & 100 & & $\mathrm{mg} / \mathrm{l}$ & 25 & 45 & 35 & 24 & 187 & 42.5 & 358.5 \\
\hline Sulphate & NS & 400 & $\mathrm{mg} / 1$ & 69.7 & 5.4 & 21 & 1 & 7.61 & & 104.71 \\
\hline Phenol & $0.05(\mathrm{dt})$ & & $\mathrm{mg} / 1$ & $<0.001$ & $<0.001$ & $<0.001$ & $<0.001$ & $<0.001$ & $<0.001$ & 0 \\
\hline Arsenic & 10 & 0.05 & $\mathrm{mg} / \mathrm{l}$ & $<0.001$ & $<0.001$ & $<0.001$ & $<0.001$ & $<0.001$ & $<0.001$ & 0 \\
\hline Barium & 50 & 0.05 & $\mathrm{mg} / \mathrm{l}$ & 2.3 & $<0.001$ & $<0.001$ & $<0.001$ & $<0.001$ & $<0.001$ & 2.3 \\
\hline Cadmium & 0.4 & 200 & $\mathrm{mg} / 1$ & $<0.001$ & $<0.001$ & $<0.001$ & $<0.005$ & $<0.005$ & $<0.002$ & 0 \\
\hline
\end{tabular}


Table Continued

\begin{tabular}{|c|c|c|c|c|c|c|c|c|c|c|}
\hline \multicolumn{4}{|l|}{ Regulatory limits } & \multirow{2}{*}{$\begin{array}{l}\text { NORKPO } \\
\text { BH- NOR }\end{array}$} & \multirow{2}{*}{$\begin{array}{l}\text { SIME } \\
\text { BH- } \\
\text { SIM }\end{array}$} & \multirow{2}{*}{$\begin{array}{l}\text { NONWA } \\
\text { BH-NOW }\end{array}$} & \multirow{2}{*}{$\begin{array}{l}\text { BOMU } \\
\text { BH-BM }\end{array}$} & \multirow{2}{*}{$\begin{array}{l}\text { OGU- } \\
\text { BOLO } \\
\text { BH-OGB }\end{array}$} & \multicolumn{2}{|c|}{ OKRIKA } \\
\hline $\begin{array}{l}\text { Analysis } \\
\text { parameters }\end{array}$ & $\begin{array}{l}\text { EGASPIN } \\
\text { Target } \\
\text { values }\end{array}$ & $\begin{array}{l}\text { WHO } \\
\text { Limit }\end{array}$ & UNITS & & & & & & $\begin{array}{l}\text { BH- } \\
\text { OKR }\end{array}$ & Total \\
\hline Chromium & 1 & 0.05 & $\mathrm{mg} / 1$ & $<0.001$ & $<0.001$ & $<0.001$ & $<0.001$ & $<0.001$ & $<0.001$ & 0 \\
\hline Cobalt & 20 & & $\mathrm{mg} / 1$ & $<0.003$ & $<0.001$ & $<0.002$ & $<0.001$ & $<0.001$ & $<0.001$ & 0 \\
\hline Copper & 15 & 1 & $\mathrm{mg} / 1$ & $<0.002$ & $<0.001$ & $<0.002$ & $<0.001$ & 0.004 & $<0.001$ & 0.004 \\
\hline Nickel & 15 & & $\mathrm{mg} / \mathrm{l}$ & $<0.001$ & $<0.001$ & $<0.002$ & $<0.002$ & $<0.002$ & $<0.001$ & 0 \\
\hline Zinc & 65 & 5 & $\mathrm{mg} / 1$ & $<0.002$ & $<0.001$ & $<0.001$ & $<0.005$ & 0.027 & $<0.001$ & 0.027 \\
\hline $\operatorname{Iron}(\mathrm{Fe})$ & 1 & 0.3 & $\mathrm{mg} / 1$ & 0.002 & $<0.001$ & 0.001 & 0.09 & 0.03 & 0.26 & 0.383 \\
\hline $\begin{array}{l}\text { Magnesium ( } \\
\text { Hardness) }\end{array}$ & NS & 150 & $\mathrm{mg} / \mathrm{l}$ & 4.3 & 0.32 & 0.001 & 0.09 & 0.02 & 2.78 & 7.511 \\
\hline Calcium ( Hardness) & NS & 200 & $\mathrm{mg} / \mathrm{l}$ & 1.5 & 2 & 1.4 & 1.02 & 3.21 & 3.11 & 12.24 \\
\hline Total Hardness & NS & 500 & $\mathrm{mg} / 1$ & 14.31 & 3.4 & 12.3 & 1.11 & 12.9 & 25.4 & 69.42 \\
\hline $\mathrm{Na}^{--}$ & NS & 200 & $\mathrm{mg} / 1$ & 13.2 & 5.5 & 18.2 & 1.2 & 7.5 & 35.6 & 81.2 \\
\hline $\mathrm{Ca}_{2}^{+}$ & NS & NS & $\mathrm{mg} / 1$ & 10.3 & 3.3 & 8.3 & 0.2 & 4 & 33.19 & 59.29 \\
\hline $\mathrm{mg}_{2}^{+}$ & N/A & NS & $\mathrm{mg} / 1$ & 4.35 & 0.07 & 0.2 & 0.08 & 0.3 & 7.29 & 12.29 \\
\hline $\mathrm{k}^{+}$ & NS & NS & $\mathrm{mg} / \mathrm{l}$ & 1.8 & 0.07 & 0.02 & 0.08 & 2.5 & 20.52 & 24.99 \\
\hline $\mathrm{HCO}_{3}^{-}$ & NS & NS & $\mathrm{mg} / \mathrm{l}$ & 28.6 & 3.2 & 0.02 & 3.05 & 1.5 & 0.0085 & 36.3785 \\
\hline $\mathrm{Cl}^{-}$ & & 250 & $\mathrm{mg} / \mathrm{l}$ & 39.8 & 10.2 & 16.4 & 22.02 & 10.2 & 48.6 & 147.22 \\
\hline $\mathrm{SO}_{4}{ }^{2-}$ & N/A & 400 & $\mathrm{mg} / \mathrm{l}$ & 3.7 & 9 & 10 & 0.26 & 24.7 & 58.42 & 106.08 \\
\hline $\mathrm{NO}_{3}^{-}$ & N/A & 10 & $\mathrm{mg} / \mathrm{l}$ & 4.2 & 1.3 & 2.3 & 1.55 & 4.1 & 4.3 & 17.75 \\
\hline
\end{tabular}


Table 4 Summary results for geotechnical parameters

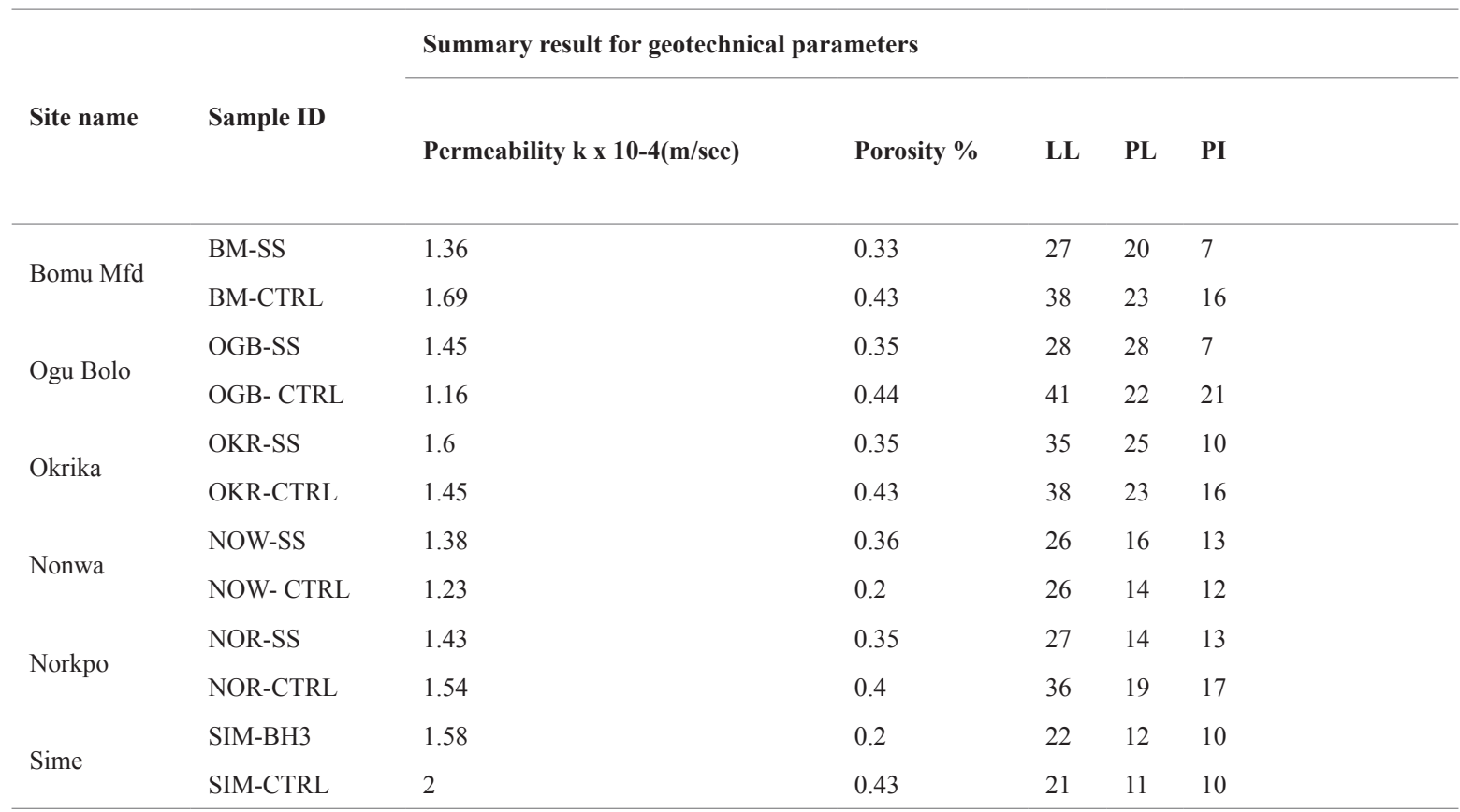

CTRL, control samples.

Table 5 Hydrocarbon utilizing bacteria/hydrocarbon utilizing fungi populations across depths of various sites

\begin{tabular}{|c|c|c|c|c|c|c|c|}
\hline \multirow[t]{3}{*}{ Site name } & \multirow[t]{3}{*}{ Sample ID } & \multirow{2}{*}{\multicolumn{3}{|c|}{$\begin{array}{l}\text { Depth } \\
\text { Hydrocarbon utilizing bacteria X } 104 \text { (cfu/g) }\end{array}$}} & \multicolumn{3}{|c|}{ Depth } \\
\hline & & & & & \multicolumn{3}{|c|}{ Hydrocarbon utilizing fungi x 104 (cfu/g) } \\
\hline & & $1 \mathrm{~m}$ & $2 \mathrm{~m}$ & $3 \mathrm{~m}$ & $1 \mathrm{~m}$ & $2 \mathrm{~m}$ & $3 \mathrm{~m}$ \\
\hline \multirow[t]{4}{*}{ Bomu Manifold } & $\mathrm{BM}-\mathrm{SS}_{1}$ & 7.1 & 6.91 & 5.24 & 4.9 & 4.68 & 3.78 \\
\hline & $\mathrm{BM}-\mathrm{SS}_{2}$ & 0 & 6.3 & 4.85 & 0 & 4.25 & 4.6 \\
\hline & $\mathrm{BM}-\mathrm{SS}_{3}$ & 0 & 6.1 & 5.65 & 0 & 4.070 & 4.03 \\
\hline & BM-SS5(CTRL) & 0 & 0 & 0 & 0 & 0 & 0 \\
\hline \multirow[t]{4}{*}{ Ogu Bolo } & $\mathrm{OGB}-\mathrm{SS}_{1}$ & 8.82 & 2.1 & 1.1 & 5.1 & 1.42 & 1.22 \\
\hline & ${\mathrm{OGB}-\mathrm{SS}_{2}}_{2}$ & 1.6 & 2.9 & 2.3 & 1.14 & 1.87 & 1.62 \\
\hline & ${\mathrm{OGB}-\mathrm{SS}_{3}}_{2}$ & 3.5 & 2.5 & 1.2 & 2.32 & 1.52 & 1.1 \\
\hline & OGB-Control & 0 & 0 & 0 & 1 & 1 & 0 \\
\hline \multirow[t]{4}{*}{ Okrika } & $\mathrm{OKR}_{-} \mathrm{SS}_{1}$ & 8.82 & 2.1 & 1.1 & 4.1 & 1.42 & 1.22 \\
\hline & $\mathrm{OKR}_{-} \mathrm{SS}_{2}$ & 1.6 & 2.9 & 2.3 & 1.14 & 1.87 & 1.62 \\
\hline & $\mathrm{OKR}_{-} \mathrm{SS}_{3}$ & 3.5 & 2.5 & 1.6 & 2.32 & 1.52 & 1.1 \\
\hline & OKR-CTRL & 0 & 0 & 0 & 1 & 1 & 0 \\
\hline \multirow[t]{4}{*}{ NONWA } & NOW-BH & 4.82 & 2.1 & 1.1 & 3.1 & 1.12 & 1.02 \\
\hline & NOW-BH 2 & 0.6 & 1.2 & 2.2 & 1.12 & 1.17 & 1.22 \\
\hline & NOW- $\mathrm{BH}_{3}$ & 2.5 & 2.2 & 1.2 & 1.12 & 1.22 & 1.15 \\
\hline & NOW-BH- CTRL & 0 & 0 & 0 & 1 & 1 & 0 \\
\hline \multirow[t]{4}{*}{ NORKPO } & NOR-BHC & 8.82 & 2.1 & 1.1 & 5.1 & 1.42 & 1.22 \\
\hline & NOR-BHD & 1.6 & 2.9 & 2.3 & 1.14 & 1.87 & 1.62 \\
\hline & NOR-BHJ & 3.5 & 2.5 & 1.6 & 2.32 & 1.52 & 1.1 \\
\hline & NOR-CTRL & 1.6 & 0.6 & 0.8 & 1 & 1 & 0.45 \\
\hline \multirow[t]{4}{*}{ SIME } & $\mathrm{SIM}_{-}-\mathrm{BH}_{1}$ & 8.82 & 2.1 & 1.1 & 0 & 0 & 0 \\
\hline & $\mathrm{SIM}_{-} \mathrm{BH}_{2}$ & 1.6 & 2.9 & 2.3 & 0 & 0 & 0 \\
\hline & $\mathrm{SIM}_{-\mathrm{BH}_{3}}$ & 0.5 & 1.3 & 1 & 1.14 & 1.87 & 1.62 \\
\hline & SIM-CTRL & 1.6 & 0.6 & 0.8 & 0 & 0 & 0 \\
\hline
\end{tabular}


The lowest degrader counts were recorded at the control samples BM-CTRL, OGB-CTRL, OKR-CTRL, NOR-CTRL and SIM-CTRL (Table 5). Moderate to normal microbial growth activity showed at $2 \mathrm{~m}$ depth samples and minimal microbial presence and growth showed at the $3 \mathrm{~m}$ depth samples with a general activity spike at BM-SS1, BMSS2, BM-SS3. This spike may be the result of favourable population growth conditions at the site necessitated by the soil agitation due to on-going remediation activity at the location. These results showed evidence of contamination of the study sites by crude petroleum and reduced conditions with increasing depths which is likely to enhance contaminant persistence in the impacted medium. Heterotrophic microorganisms carry out decomposition of organic matter and recycle elements to improve soil fertility. ${ }^{25}$ Petroleum degrading microorganisms occur in nature and increase in response to crude oil contamination; however, during recovery after spillage, the numbers of hydrocarbon utilizers' return, at most sites, to background levels as the oil disappears due to biodegradative removal. ${ }^{26}$ Microbial growth/ activity is normal in samples that had high counts of hydrocarbon utilizing bacteria and fungi while the control and less contaminated samples showed reduced microbial growth/activity. Microbiological results observed suggest that the study area has been exposed by chronic crude oil presence over time. Natural environments such as soils can recover from crude oil contamination due to presence of petroleum degrading microorganisms in nature; the number of degraders may return to background levels as the oil disappears due to biodegradative removal.

\section{Porosity}

Given the effective porosities of between $0.2 \%-0.3 \%$ of the silty sand bands, the downward mobilisation of contaminant will be vertically high. The mean annual rainfall of the nearest weather station is $2438 \mathrm{~mm} /$ annum. The high infiltrating and percolating water in the area which are dependent on precipitation and magnified by a lack of dispersion by water drainage channels increases the risk of groundwater contamination. This takes into account the fact that the transport of contaminants in groundwater is dependent on the solubility of the substance in water and advective flow, retardation and mechanical dispersion of the solute which are dependent on the geophysical properties of the soil in a specific location. Porosities and permeability values at impacted sites are generally lower than control sites. Permeability at BM-SS is lower than BM-CTRL. This may be as a result of the much soil agitation due to on-going remediation works at the time of the investigation. The impacted sections of the site have suffered some distortions and so is the soil profile. There is a generally slight reduction in porosity values at the impacted sites compared with the control sites. These reductions may be attributed the effects of the crude oil contamination. The control sites BM-CTRL, OKR-CTRL, NOR-CTRL and SIM-CTRL showed higher permeability values than impacted sites. These changes in soil characteristics have the capability of determining the behaviour of contaminants in the soil.

\section{Soil particle sizedistribution/consistency limits}

The grain sizes of soils are poorly graded from fine sands of $0.07 \mathrm{~mm}$ sieve sizes to fine gravel sizes of $4 \mathrm{~mm}$ sieve sizes (Table 4). This grading is typical of beach sand types. The grain distribution increases as one moves towards the sea from Norkpo to Okrika indicating that the ease of contaminant infiltration into the subsurface will as well increase in that order owing to increasing permeability of the soil. The soil profile across the study area grade from fine silty sands to fine gravel sand. The soil mixtures were as varied across the sites. However, the soil profile up to depth of investigation is generally dominated by silts, sands and sandy clay in different proportions. This kind of soil will not be able to stop infiltration of liquid hydrocarbon product and movement of contaminated water through it will continue unhindered. Figure 5 shows the generalised groundwater flow direction. The Atterberg limits are generally lower in the impacted samples. Liquid limit values for impacted soils range from 22-35 and control sites values is between 21-41; Plastic limit is 12-28 for impacted and 11-23 for control, Plasticity index is 7-13 for impacted and 10-16 for control sites. This is an indication of alteration due to the presence of contaminants in the impacted samples. Figure 6 shows the organic contaminant distribution while Figure 7 shows total petroleum hydrocarbon. Figure 8 depicts the Poly Aromatic Hydrocarbon concentration trend across the area while Figure 9 is the chart showing the soil permeability, porosity and consistency. Figure 10 is a Typical Soil Particle Size Distribution for Okrika while Figure 11 is the Soil Profile Across the study area. Figure 12 is the EGASPIN Intervention and Target level disparity for TPH. ${ }^{27-30}$

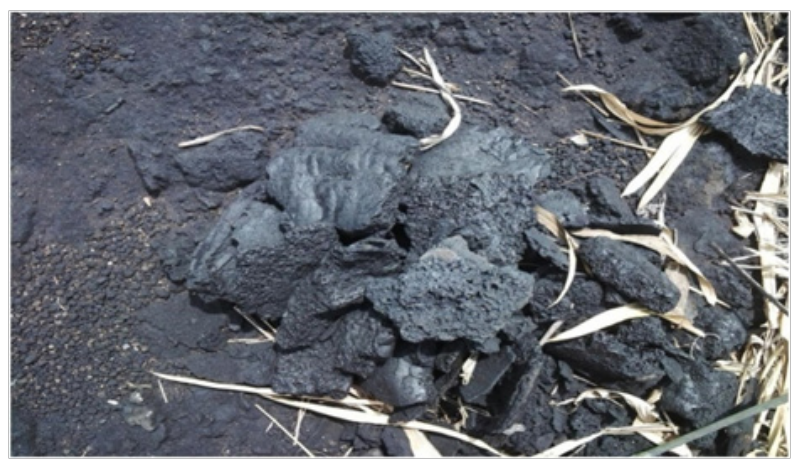

Plate I Completely burnt carbonized material at artisanal refining site @ Okrika.

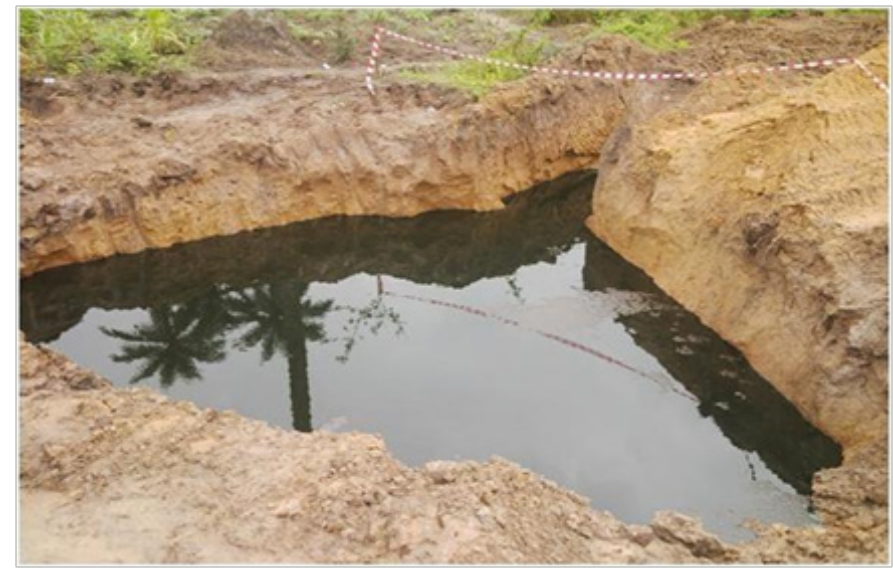

Plate 3 Pool of oil welling up in an open excavation at a remediation site @ Ogale 


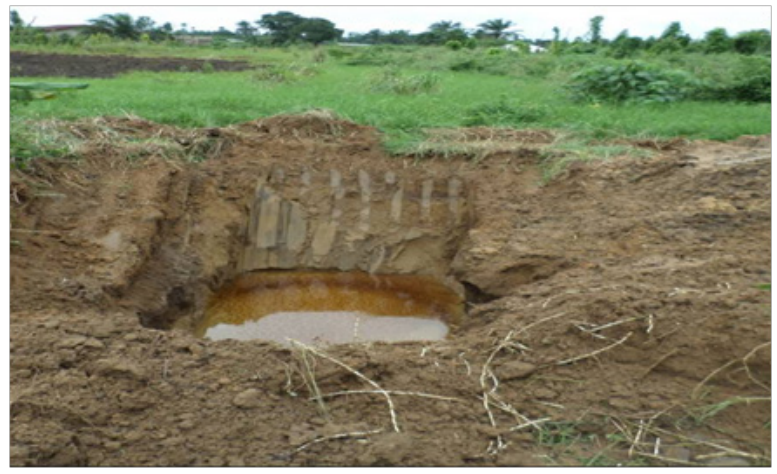

Plate 4 Typical shallow groundwater contamination impact @ Bomu area.

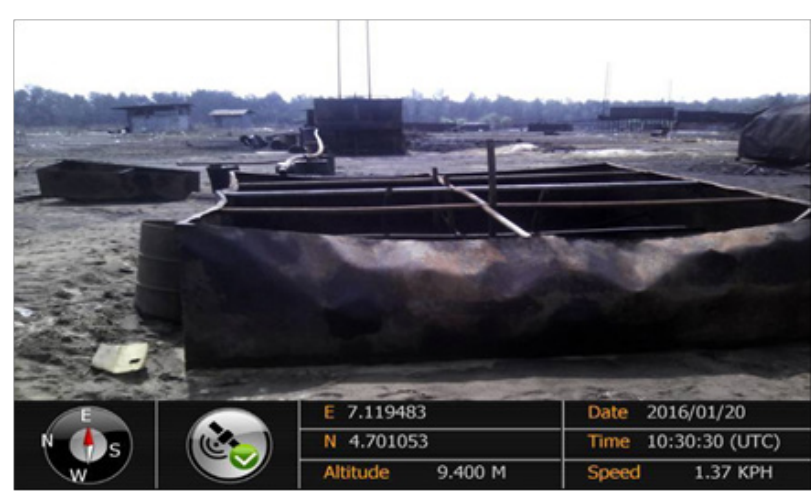

Plate 5 Environmental contamination resulting from illegal crude oil refining @ Okrika.

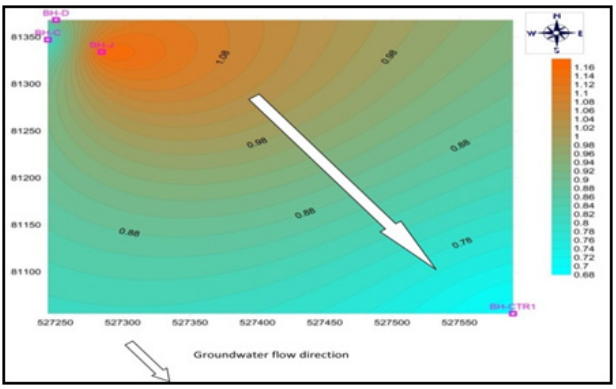

Figure 5 Generalised groundwater flow direction.

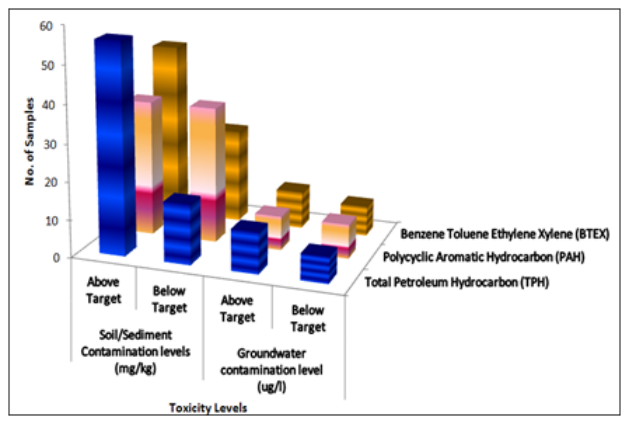

Figure 6 Organic contaminant distribution.

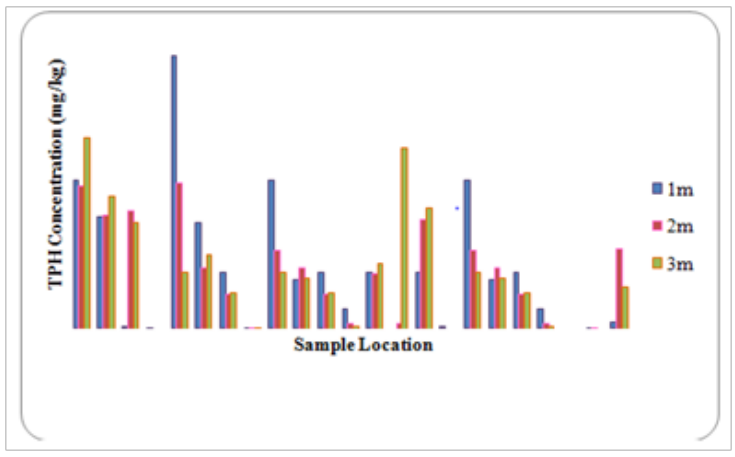

Figure 7 Total petroleum hydrocarbon.

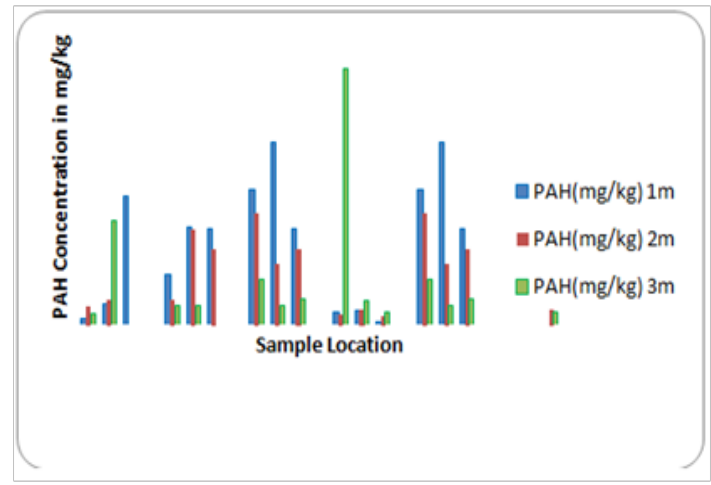

Figure 8 Poly aromatic hydrocarbon concentration trend across the area.

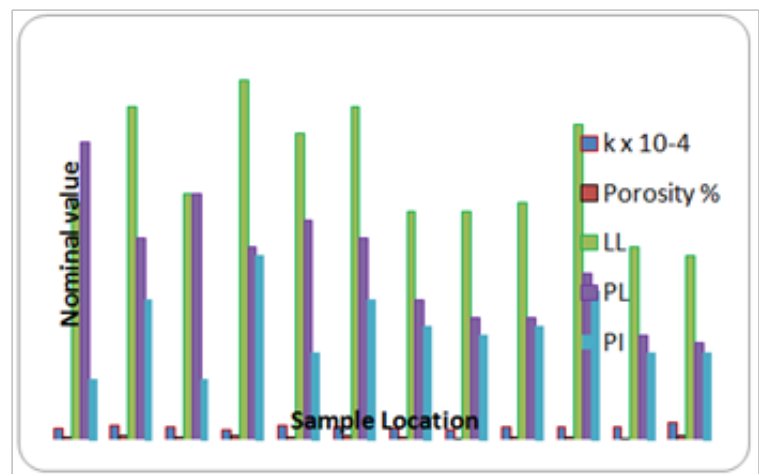

Figure 9 Soil permeability, porosity and consistency chart.

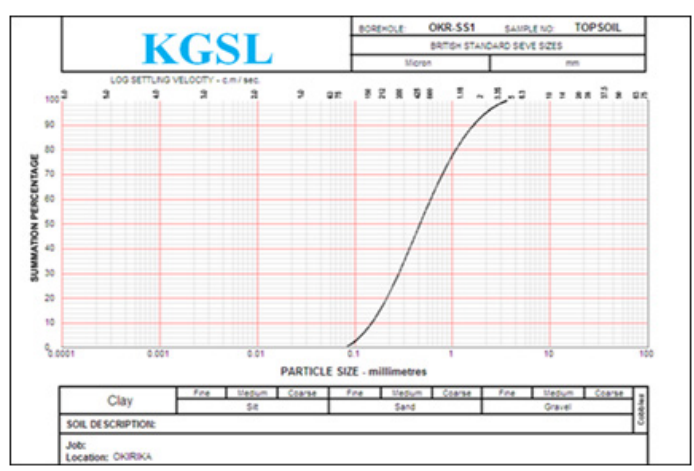

Figure 10 Typical soil particle size distribution for Okrika.

Citation: Nwankwoala HO, Mzaga TM. Geo-environmental assessment of hydrocarbon contaminated sites in parts of central swamp depobelt, Eastern Niger delta. MOJ Eco Environ Sci. 2017;2(3): I00-II2. DOI: 10.15406/mojes.2017.02.00023 


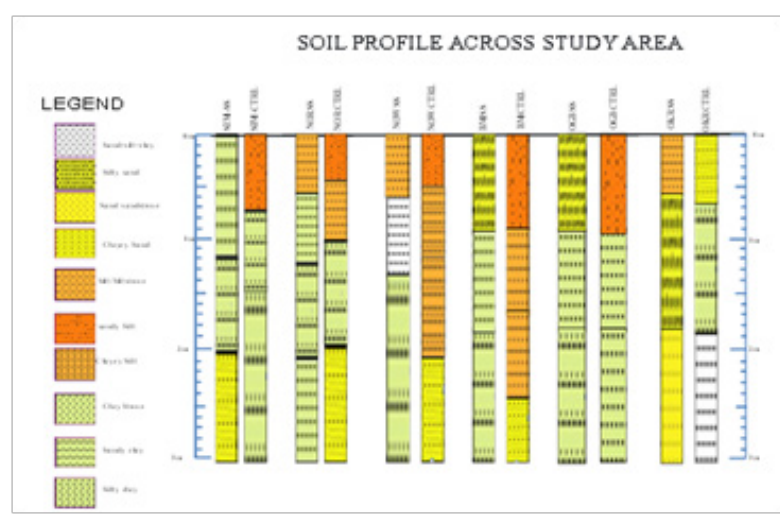

Figure II Soil profile across the study area.

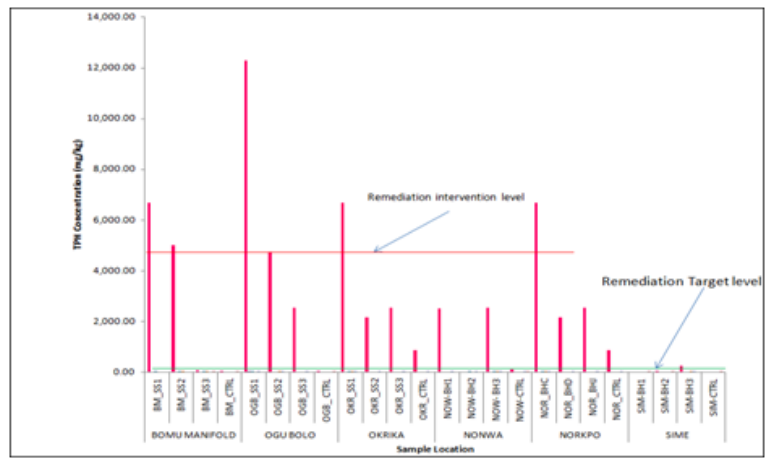

Figure 12 EGASPIN intervention and target level disparity for TPH

\section{Conclusion}

The results of this study revealed that most of soil and shallow groundwater in the area contain significantly high hydrocarbon contaminants concentrations. The predominantly silt sand and clay surficial geology of the study area is a major determinant of the extent to which contaminants can infiltrate the ground and percolate downwards. This study has also revealed that a combination of factors including porous soil structure, relatively light and soluble nature of the Nigerian crude oil, high rainfall, dynamic environment and continuous dosing of the environment with crude oil products from incessant spills, artisanal refining activities has a potential to allow widespread migration of crude oil products from spill sources into rivers, creeks, land and the groundwater resources. The current understanding of risk posed by hydrocarbon contamination is a wakeup call to government agencies and other relevant stakeholders to urgently take proactive steps to reviewing of relevant legislation, enforcement and pollution management processes that will be protective of humans, soil, plants, surface and groundwater resources.

\section{Acknowledgements}

None.

\section{Conflict of interest}

The author declares no conflict of interest.

\section{References}

1. Giadom FD, Akpokodje EG, Tse AC. Modeling contaminant migration patterns of non aqueous phase liquids in the Niger delta region. Journal of environmental earth sciences. 2015.

2. Nwankwoala HO, Ngah SA. Salinity Dynamics: Trends and vulnerability of aquifers to contamination in the Niger Delta. Comprehensive Journal of Environmental and Earth Sciences. 2013;2(2):18-25.

3. Olof L, Jonas P. Oil contamination in ogonil and Niger delta. Journal AMBIO. 2013;42(6):685-701.

4. Clay SL. Fate of petroleum hydrocarbons in the environment. Unpublished MSc dissertation submitted to McMaster University, School of geography and earth sciences; 2014.

5. Giadom FD, Tse AC. Groundwater contamination and environmental risk assessment of a hydrocarbon contaminated site in Eastern Niger Delta, Nigeria. Journal of Environment and Earth Sciences. 2014;5(14).

6. Devaull G, Truchon. Conceptual site models for Niger Delta Ecozones. Shell global solutions international BV Rijswijk. SR.15.10103 .Available online@ shell global information centre; 2015.

7. Amangaraba GT, Njoku JD. Asessing groundwater vulnerability to the activities of artisanal refining in Bolo and environs, Ogu Bolo local government area of rivers state; Nigeria. British Journal of environment and climate change. 2012;2(1):28-36.

8. Osuji LC, U Opiah. Hydrocarbon contamination of a terrestrial ecosystem: the case of Oshire-2 oil spill in Niger Delta, Nigeria. The Environmentalist. 2007;27(3):337-340.

9. Akpokodje EG. The engineering-geological characteristics and classification of the major superficial soils of the Niger Delta. Engineering Geology. 1987;3:193-211.

10. Osuji LC, Iruka N. An appraisal of the impact of petroleum hydrocarbons on soil fertility: The Owazaexperience. African Journal of Agricultural research. 2006;2(7):318-324.

11. Odigi MI. Evaluating groundwater supply in the eastern Niger delta Nigerian Journal of Mining Geology. 1989;25:159-164.

12. Short KC, Stauble AJ. Outline geology of the Niger delta. American Association of Geologists.1967;51(5):761-779.

13. Nwankwoala HO, Marshal HI, Oborie E. Characterization and quantitative indicators of ground water quality in Okrika, Rivers State, Nigeria. International Journal of Science Inventions today. 2014;2(4):319-334.

14. United Nations Environmental Programme. Environmental Assessment of Ogonil; 2011.

15. Nwankwoala HO, Ngah SA. Assessing chloride distribution and concentrations in the aquifer systems of the Niger Delta. Journal of Geosciences and Geomatics. 2015;3(5):128-132.

16. Ngah SA, Nwankwoala HO. Evaluation of geotechnical properties of the sub-soil for shallow foundation design in Onne, Rivers State, Nigeria. The Journal of Engineering and Science. 2013;2(1):8-16.

17. Etu-Efeotor JO. Preliminary Hydrogeochemical investigations of SubSurface waters in parts of the Niger Delta. Nigerian Journal of Mining Geology. 1981;18(1):103-105.

18. Amadi PA, Morrison T, Ofoegbu CO. Hydrogeochemical assessment of groundwater quality in parts of the Niger Delta, Nigeria. Environmental Geology and Water Science. 1989;14(3):195-202. 
19. Weber KJ, Daukoru EM. Petroleum geology of the Niger delta: 9th World Petroleum Congress Proceedings. 1975;2:209-221.

20. Nwankwoala HO, Amadi AN, Oborie E, et al. Hydrochemical factors and correlation analysis in groundwater quality in Yenagoa, Bayelsa State. Nigeria Journal of Applied Ecology and Environmental Sciences. 2013;2(4):100-105.

21. Ngerebara OD, Nwankwoala HO. Groundwater potentials in the offshore Niger Delta environment, Nigeria. Electronic Journal of Environmental Hydrology. 2008;16:28.

22. Etu-Efeotor JO, Odigi MT. Water supply problems in the eastern Nige Delta. Bulletin Nigeria Mining and Geoscience Association. 1983;20:183 1992.

23. Amadi AN, Olasehinde PI, Yisa J, et al. Geostatistical assessment of groundwater quality from coastal aquifers of eastern Niger Delta, Nigeria. Journal of Geosciences. 2012;2(3):51-59.

24. United states environmental protection agency. National primary drinking water regulations; 2009.
25. Torstensson L, Pell M, Stenberg B. Need of a strategy for evaluation of arable soil quality. Journal of Human Environment. 1998;27(1):4-8.

26. Atlas RM. Microbial degradation of petroleum hydrocarbons: an environmental Perspective. Microbiological Review. 1981;45(1):180-209.

27. EGASPIN. Environmental guidelines and standards for the petroleum industry in Nigeria. Issued by the department of petroleum resources. Lagos: Revised Edition; 2002.

28. Etu-Efeotor JO, Akpokodje EG. Aquifer systems of the Niger Delta Journal of Mining Geology. 1990;26(2):279-284.

29. Shell petroleum development company limited. Oil spill response and remediation management standards (OSRRMS); 2001.

30. Zihms SG, Switzer C, Karstunen M, et al. Understanding the effects of high temperatures on the engineering properties of soil : Lessons to share from smouldering remediation experience. Flamma. 2013;6(1):5-7. 